\title{
Frontières
}

\section{Les femmes souffrantes de par le monde}

\section{Monique Dumais}

Volume 17, numéro 2, printemps 2005

Surtout, ne pas souffrir

URI : https://id.erudit.org/iderudit/1073497ar

DOI : https://doi.org/10.7202/1073497ar

Aller au sommaire du numéro

Éditeur(s)

Université du Québec à Montréal

ISSN

1180-3479 (imprimé)

1916-0976 (numérique)

Découvrir la revue

Citer ce document

Dumais, M. (2005). Les femmes souffrantes de par le monde. Frontières, 17(2), 96-99. https://doi.org/10.7202/1073497ar d'utilisation que vous pouvez consulter en ligne.

https://apropos.erudit.org/fr/usagers/politique-dutilisation/ 


\title{
Les femmes souffrantes de par le monde
}

\author{
Monique Dumais, Ph.D., \\ professeure retraitée de I'Université du Québec à Rimouski.
}

«Les souffrantes»

par Yves Simon, écrivain et chanteur

Aux femmes afghanes

Derrière des grilles nommées tchadris

Des femmes sont prisonnières,

des femmes d'aujourd'hui

Héroïnes au secret d'une histoire délirante,

Ce sont elles les souffrantes.

Kaboul maboule

Les masques foulent

Lintégrité et la beauté

Des amantes, des filles, des femmes, de souffrantes

Notre moitié.

Où sont les yeux des Afghanes

Elles regardent ailleurs et pleurent

Où sont leurs visages? Elles regardent ailleurs

Vers l'intérieur.

Des chars de guerre ont défilé

Des hommes vainqueurs, inquisiteurs d'intimité

Qui ont foulé, défiguré... charge infamante

Le rêve sacré des souffrantes.

Où sont les yeux des Afghanes

Elles regardent ailleurs et pleurent

Où sont leurs visages? où sont leurs visages?

Les kalachnikovs sur la hanche

Les hommes paradent et se déhanchent

Recouvrant de voiles sombres et de pénombre

Les rêves infinis des souffrantes

Derrière des grilles nommées tchadris

Des femmes sont prisonnières, des femmes d'aujourd'hui

Héroïnes au secret d'une histoire délirante

Ce sont elles les souffrantes.

Où sont les yeux des Afghanes

Où sont les yeux des Afghanes ${ }^{1}$

(Simon, 2002, p. 15-16)
Ce texte figure parmi les cent lettres écrites pour les femmes afghanes; elles ont été publiées dans la revue L'Humanité, un numéro hors série paru en septembre 2001. Ces lettres se veulent solidaires des détresses, des douleurs et aussi des espoirs des femmes en Afghanistan après la guerre.

Les souffrances des femmes prennent des proportions immenses avec la mondialisation. Elles s'inscrivent dans le marché qui est au centre de ce phénomène dirigé par le néolibéralisme, tout particulièrement dans ce marché du sexe où les femmes deviennent de pures marchandises. Aucune catastrophe n'arrête l'appétit effréné de l'argent, au contraire, les pires événements suscitent de nouvelles occasions pour les profiteurs de se livrer aux transactions les plus funestes (Dusch, 2002; Wichterich, 1999, p. 100-108). Ainsi, a-t-on lu dans les journaux, après la catastrophe des tsunamis que les pays de l'Asie du Sud-Est donnent libre cours au trafic sexuel.

Rappelons que les populations des pays durement touchés aujourd'hui par les tsunamis souffrent depuis longtemps d'un autre fléau, le trafic sexuel, lié au tourisme sexuel. Dans des pays comme la Thailande, le Sri Lanka, l'Indonésie, la Malaisie et l'Inde, l'industrie du sexe attire chaque année des millions de visiteurs étrangers vers cette région devenue un paradis pour les pédophiles du monde entier. Les bordels et les boîtes de nuit pullulent dans la région. Ils offrent au menu des femmes et des enfants de tous âges, souvent arrachés à leurs parents ou vendus par un proche, pour satisfaire tous les fantasmes des hommes venus des quatre coins du monde profiter du laxisme ambiant. (Gedeah, 2005)

Je m'arrêterai surtout à scruter cette brisure sociale qu'est la réalité brutale du trafic des femmes, en tentant de montrer les 
berceau tragique

effets destructeurs qu'elle exerce sur la vie individuelle des femmes et sur la vie sociale en général, ce qui, par conséquent, nous conduit à un mal généralisé à l'humanité.

\section{UNE RÉALITÉ BRUTALE}

«Vendues par leur famille, kidnappées, ou rêvant tout simplement d'une nouvelle vie dans un pays occidental, environ quatre millions de femmes et d'enfants basculent chaque année dans l'enfer de la traite à des fins de prostitution»(Cauchy, 2004). Le trafic sexuel, cette forme d'approvisionnement du système prostitutionnel, est une réalité qui n'est pas nouvelle, ce qui ne la rend pas excusable: «les femmes ont été trafiquées dans la Mésopotamie d'il y a 5000 ans », mais avec la mondialisation, la mise en vente des corps a pris des dimensions fulgurantes. "Le corps des femmes et des enfants - et de plus en plus celui des hommes - est devenu un "produit" bradé sur le marché mondial, une matière première à exploiter, soumise aux lois du commerce international» (Legardinier, 2002, p. 4).

Comment définir le trafic sexuel? La définition élaborée par le groupe de recherche-action sur le trafic sexuel de femmes au Québec, sous la responsabilité de Marie-Andrée Roy et Lyne Kurtzman², est très utile.

Le trafic sexuel désigne le recrutement, le transport, à l'intérieur et à l'extérieur d'un pays, le transfert, l'hébergement ou l'accueil de personnes dans le but de les obliger à exercer des activités dans l'industrie du sexe ou à se prostituer. Il procède de différentes façons pour parvenir à des visées d'exploitation sexuelle: la menace de recours, ou le recours à la force, l'enlèvement, la fraude, la tromperie, l'abus d'autorité ou d'une situation de vulnérabilité ou encore l'offre ou l'acceptation de paiements ou d'avantages. Le trafic peut parvenir à ses visées en obtenant le consentement d'une personne ayant autorité sur une autre aux fins d'exploitation. Le consentement d'une victime de trafic sexuel est indifférent lorsque l'un des moyens ci-haut mentionnés a été utilisé. Le trafic sexuel des femmes provoque des mouvements transfronta-

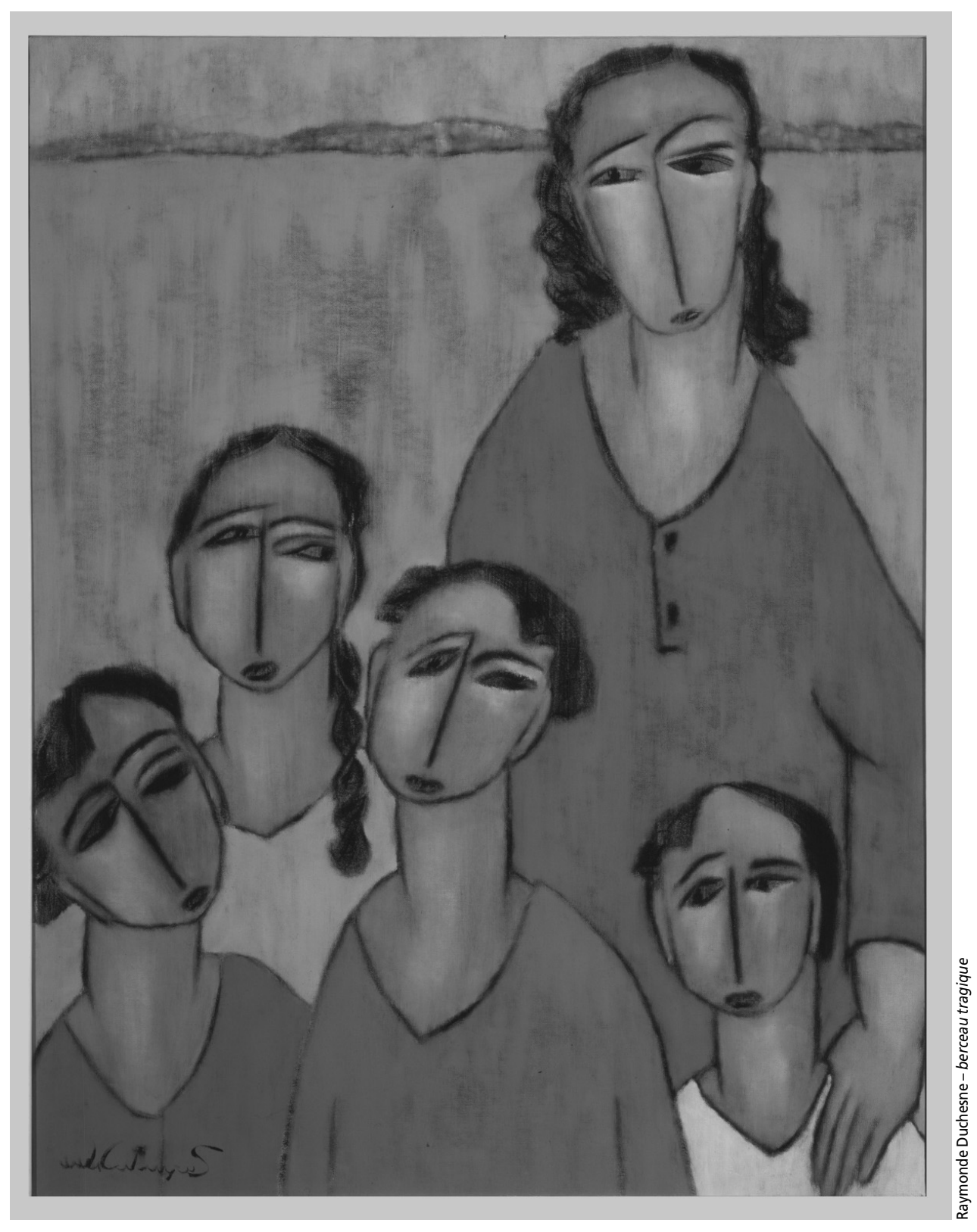

liers de personnes entre pays marqués par une inégalité économique.

(Roy, 2005, p. 30).

Cette définition mentionne les différentes circonstances aggravantes de cette réalité qui est réellement brutale et dégradante pour tout être humain pris dans cet engrenage. Le trafic des humains résulte de facteurs structurels qui le rendent possible: ils sont d'ordre économique, politique, culturel, religieux.

Tout d'abord s'inscrivent les facteurs économiques avec la loi du profit du marché néolibéral où les corps sont rendus disponibles pour répondre à l'industrie du sexe, notamment à celle du tourisme sexuel. C'est le règne de la consommation qui s'étend sous ses formes les plus avilissantes pour les êtres humains. Les corps sont effectivement objets de vente: selon le rapport 2000 des Nations Unies pour la population, quatre millions de femmes et de fillettes sont vendues chaque année dans le monde à de futurs époux, à des proxénètes ou à des marchands d'esclaves.

Au plan politique, il apparaît clairement que «de plus en plus d'États semblent désireux d'intégrer les revenus de la prostitution dans le P.I.B.» (Legardinier, 2002, p. 28). Il ne s'agit pas seulement de certains pays d'Asie du Sud-Est, mais aussi de pays comme l'Allemagne et les Pays-Bas, qui, en légalisant une partie du proxénétisme, montrent les intérêts financiers qu'ils reconnaissent dans le marché des corps. D'autres pays délivrent de façon douteuse des visas pour des activités dites artistiques. Le Canada est touché par cette problématique vaste et complexe si bien que le gouvernement a décidé de mettre sur pied le Groupe de travail interministériel sur la traite des personnes (GTITP) regroupant 
des représentants du ministère de la Justice, de la Gendarmerie royale du Canada, de Condition féminine Canada et de Citoyenneté et immigration Canada (Roy, 2002, p. 31).

Du côté culturel, le statut infériorisé des femmes dans plusieurs pays ouvre les portes au trafic sexuel. "Sur 110 millions d'enfants non scolarisés dans le monde, les deux tiers sont des filles. Partout dans le monde, elles sont les premières touchées par l'analphabétisme, l'accès réduit à la scolarité et à l'emploi, l'insuffisance de formation, le sexisme du marché du travail» (Legardinier, 2002, p. 32). La recherche d'emploi devient ainsi un guet-apens pour une majorité de femmes pauvres qui veulent se sortir de leur situation misérable. À ce sujet, Christa Wichterich raconte l'histoire d'Anna, une femme polonaise qui s'est retrouvée prise dans les filets du trafic international.

Sur un panneau d'affichage dans sa ville natale de Szczecin, en Pologne, elle a lu en 1993 une offre d'emploi d'été comme auxiliaire de maison. Elle a travaillé une brève période comme aide-cuisinière, et on lui a rapidement promis un emploi analogue à Berlin. Là, elle a été forcée à se prostituer. On la battait lorsqu'elle protestait. Elle était constamment surveillée, et on lui amenait des clients toutes les nuits. Ses geôliers avaient gardé ses papiers d'identité. Finalement vendue au café Casablanca, elle touchait 35 francs par passe. Les propriétaires de maisons closes berlinoises ou ouest-allemandes paient entre 10000 et 17000 francs pour des femmes comme Anna, selon leur attrait. Une voiture volée en rapporte 35000 .

(Wichterich, 1999, p. 101.)

Machisme et sexisme se combinent pour la domination des femmes par des hommes qui sont des consommateurs payeurs sur fond d'une libération sexuelle où il faut satisfaire ses impulsions sexuelles. Ainsi, le sexe devient culturellement banalisé par différents moyens pornographiques tels que revues, vidéos, notamment des sites Internet. Nous sommes dans une «culture d'exaltation du sexe et de la pornographie» (Roy, 2005, p. 32) avec tous les effets pervers qui s'ensuivent.

Le facteur religieux s'ajoute aux trois précédents. Des traditions religieuses maintiennent les femmes dans un statut de seconde zone et ne présentent pas une vision positive de la sexualité. Les femmes sont encore perçues en dépendance des hommes, comme d'éternelles mineures qui doivent se soumettre aux diktats de leurs supérieurs.

\section{DES EFFETS DESTRUCTEURS}

Le rêve brisé de Cendrillon ${ }^{3}$, un Grand reportage de RDI, a montré le destin tragique de quelques-unes des 500000 femmes de l'Europe de l'Est qui sont devenues malgré elles des esclaves sexuelles. Ce qui est bouleversant, c'est de constater chez les femmes prises dans le trafic international la perte de toute identité personnelle, y compris du passeport. Elles sont soumises à différents sévices: «condamnées au silence, en situation irrégulière, sans papiers, menacées par les souteneurs, harcelées par la police, pourchassées, expulsées. Vouées à la drogue - pour tenir -, elles sont touchées par le VIH, qui connaît une progression alarmante dans certains pays » (Legardinier, 2002, p. 13). Le trafic sexuel est loin d'être un lieu de plaisir pour les femmes, un lieu d'émancipation comme certaines personnes le prétendent.

\section{LES CORPS}

\section{SONT RENDUS DISPONIBLES}

\section{POUR RÉPONDRE}

\section{À L'INDUSTRIE DU SEXE.}

On peut réellement affirmer que «les enjeux sont immenses puisqu'il s'agit de la vie et du respect des droits humains fondamentaux de millions de personnes, principalement des femmes et des enfants » (Roy, 2005 , p. 30). La priorité de la surconsommation détruit en profondeur l'intégrité des femmes et des enfants qui est touchée dans sa profondeur par le processus de surconsommation. Comme le dit Claire Lejeune (1998, p. 148), poète et prophète pour aujourd'hui: "Tuer le désir de présence à soi-même pour lui substituer le besoin insatiable de surconsommer les produits de la surproduction: n'est-ce pas l'ultime objectif du Grand Marché? Le désir de faire corps avec soi serait-il l'ennemi mortel de la "pensée unique"?»

\section{NOUS AVONS MAL À L'HUMANITÉ}

La Convention sur l'élimination de toutes les formes de discrimination à l'égard des femmes (1979) engageait tous les États signataires à «prendre toutes les mesures appropriées, incluant la législation, pour éliminer toutes les formes de traite des femmes et l'exploitation des femmes par la prostitution» (article 6), et pourtant le chemin de cette élimination totale semble encore bien long. Devant les dangers menaçants après les tsunamis, Yolande Gedeah n'a pas manqué d'affir- mer: «Des millions de femmes et d'enfants sont ainsi destinés à servir de fast food du sexe pour des touristes en mal d'exotisme. Cette situation est à l'origine d'une déstructuration profonde du tissu social» (Gedeah, 2005). En effet, des sociétés entières sont à risque. "Le système prostitutionnel demeure à l'échelle mondiale un miroir en creux des relations inégalitaires entre hommes et femmes, adultes et enfants, pays riches et pays pauvres.» (Legardinier, 2002, p. 5.)

Le problème du trafic sexuel des femmes est devenu une affaire publique tant dans les journaux que sur nos écrans de télévision. Il nous pousse à une prise de conscience aiguë des maux qui envahissent nos sociétés. Ivone Gebara qui a écrit un livre intitulé Le mal au féminin n'hésite pas à affirmer: «il y a une maladie dans nos rapports sociaux et tous et toutes nous sommes victimes de sa contagion et reproducteurs/reproductrices de cette même maladie»(Gebara, 1999, p. 115).

Cependant devant les différentes formes $\mathrm{du}$ mal, nous, en tant que citoyennes, citoyens, prenons-nous vraiment le temps de penser, de chercher des voies de sortie, de responsabilisation? Hannah Arendt, philosophe juive, a réfléchi sur ce qui s'était passé pendant la Shoah où six millions de Juifs ont été exterminés. Lors du procès d'Eichmann à Jérusalem, elle avait évoqué «la banalité du mal», ce qui n'avait pas manqué d'étonner. Quelques années plus tard, elle a expliqué ce qu'elle avait voulu signifier par là.

J'avais parlé de «la banalité du mal», entendant par là non pas une théorie ou une doctrine mais quelque chose de tout à fait factuel, un phénomène de forfaits commis à une échelle gigantesque et impossibles à rattacher à quelque méchanceté particulière, à quelque pathologie ou conviction idéologique de l'agent, lequel se distinguait peut-être uniquement par une extraordinaire superficialité

(Arendt, 1996, p. 25.)

Une extraordinaire superficialité... c'est un verdict qui pourrait tout autant s'appliquer à l'époque actuelle. Hannah Arendt avait trouvé chez Eichmann «une curieuse et authentique inaptitude à penser» (Arendt, 1996, p. 26). Cette inaptitude est encore très présente dans notre monde et nous porte à agir sans réfléchir aux conséquences de nos actions. La pente d'une grande permissivité et d'un consumérisme exacerbé entraîne l'humanité dans des engrenages de dégradation et de destruction des êtres humains.

Des actions s'imposent tant sur le plan économique, politique que culturel. La Charte mondiale des femmes pour l'humanité 


\section{LES CORPS DES FEMMES SONT DEVENUS}

\section{UNE MARCHANDISE ALIÉNÉE DE TOUTE FORME D'HUMANITÉ.}

qui est lancée à travers le monde à l'occasion du 8 mars 2005 est significative à cet égard. Elle contient plusieurs indications pour un changement de société: elle réclame un monde qui se fonde sur les valeurs d'égalité, de liberté, de solidarité, de paix et de justice. Voici quelques affirmations que j'ai retenues comme confrontantes pour le trafic sexuel des femmes.

\section{Égalité Affirmation 3}

Aucune coutume, tradition, religion, idéologie, aucun système économique ne justifie l'infériorisation de quiconque et n'autorise des actes qui remettent en cause la dignité et l'intégrité physique et psychologique.

\section{Liberté Affirmation 1.}

Tous les êtres humains vivent libres de toute violence. Aucun être humain n'appartient à un autre. Aucune personne ne peut être tenue en esclavage, subir le travail forcé, être objet de trafic, d'exploitation sexuelle, de prostitution.

\section{Solidarité Affirmation 1.}

Tous les êtres humains sont interdépendants. Ils partagent le devoir et la volonté de vivre ensemble, de construire une société généreuse, exempte d'oppression, d'exclusions, de discriminations, d'intolérance et de violences.

\section{Justice Affirmation 3.}

L'intégrité physique et morale de toutes et de tous est garantie. La peine de mort est abolie. La torture, les traitements humiliants et dégradants sont interdits. Les agressions sexuelles, les violences spécifiques à l'égard des femmes et le trafic sexuel sont considérés comme des crimes contre la personne et contre l'humanité.

Paix [...] Pour que ce monde existe, les conditions suivantes doivent être remplies: 1. des lois sont édictées et effectivement appliquées qui interdisent et condamnent toutes les formes de violences, particulièrement les violences visant spécifiquement les femmes, qu'elles soient d'ordre privé ou public, qu'elles se déroulent en temps de paix ou en temps de guerre. $[\ldots]$
Ces grandes lignes de pensée et d'action s'affichent comme des moyens de retrouver le goût de vivre notre humanité, de sortir du gouffre de l'esclavage et de la destruction systématique des êtres humains.

Une voix dans Rama s'est fait entendre, des pleurs et une longue plainte:

c'est Rachel qui pleure ses enfants et ne veut pas être consolée,

parce qu'ils ne sont plus. (Évangile selon Matthieu 2, 18) ${ }^{4}$

Des temps anciens de la Bible jusqu'à nos jours, les femmes souffrent des malheurs qui assaillent leurs enfants et elles-mêmes. Rachel, épouse de Jacob, mère des Israélites du Nord (Livre de la Genèse), pleure sur ses enfants exilés; c'est sa douleur qui est entendue. Aujourd'hui, la sculpture Femme crucifiée $^{5}$, située dans un jardin du campus de l'Université de Toronto, montre à tous les regards que les femmes ne sont pas seulement objets de plaisir mais qu'elles portent dans leur chair d'immenses souffrances. Denise Lamarche a écrit un livre Des femmes aussi portent la croix; elle offre une des quatorze stations du chemin de la croix à chacune des quatorze jeunes femmes assassinées, le 6 décembre 1989, à l'École polytechnique de l'Université de Montréal (Lamarche, 2001).

Sur cette planète dite mondialisée, le trafic sexuel des femmes m'est apparu très révélateur de tout un poids de souffrances. Objets de plaisir, les corps des femmes sont devenus une marchandise aliénée de toute forme d'humanité. Les statistiques de ce marché des corps sont accablantes et exigent une sortie de notre indifférence et de notre passivité. Ce monde de souffrances ne doit pas complètement nous anéantir. Dans Désir d'humanité, Riccardo Petrella nous invite à continuer de rêver:

Personne n'arrêtera les êtres humains de «rêver» un monde meilleur, même si tous les «rêveurs» savent que la construction d'un monde meilleur ne sera jamais définitive et qu'il y aura toujours un mieux possible au-delà des confins atteints à un moment donné dans un lieu donné. Le rêve est vie. Il est aussi indispensable à la vie que l'eau, l'air, l'amour. (Petrella, 2004, p. 16).

Et ce rêve d'une humanité meilleure, plus libre, permettant aux êtres humains de s'accomplir, je le fais intensément pour toutes les femmes du monde.

\section{Bibliographie}

ARENDT, Hannah $(1996 ; 1971)$. Considérations morales, Paris, Payot \& Rivages.

CAUCHY, ClairAndrée (2004). «Importexport de chair fraîche ", Le Devoir, vol. XCV, $\mathrm{n}^{\mathrm{o}} 256,10$ novembre, $\mathrm{p}$. A1 et A8.

DUSCH, Sabine (2002). Le trafic d'êtres humains, Paris, Presses universitaires de France, coll. «Criminalité internationale ».

GEBARA, Ivone (1999). Le mal au féminin. Réflexions théologiques à partir du féminisme, Paris/Montréal, L'Harmattan.

GEDEAH, Yolande (2005). "Aux pays des tsunamis, le trafic sexuel sévit», Le Devoir, 11 janvier, p. A7.

LAMARCHE, Denise (2001). Des femmes aussi portent la croix, Montréal, Fides.

LEGARDINIER, Claudine (2002). Les trafics du sexe. Femmes et enfants marchandises, Toulouse, Éditions Milan (Les Essentiels Milan).

LEJEUNE, Claire, (1998). Le livre de la mère. Avin/Hannut (Belgique), Éditions Luce Wilquin, coll. «Hypatie».

PETRELLA, Riccardo (2004). Désir d'humanité. Le droit de rêver, Montréal, Écosociété.

ROY, Marie-Andrée (2005). «Le trafic sexuel», Relations, janvier-février, p. 30-33.

SIMON, Yves (2002). Dans Cent lettres pour les femmes afghanes, Paris, Éditions Quai de Seine.

WICHTERICH, Christa (1999), La femme mondialisée, essai traduit de l'allemand par Olivier Mannoni, Arles, Solin, Actes Sud.

\section{Notes}

1. Cette chanson contenue dans l'album Intempestives (Barclay) est sortie en septembre 1999. Le texte en a été traduit en persan (Afghanistan) par l'écrivain afghan en exil à Paris, Atiq Rahimi, afin d'être publié dans des revues et journaux pakistanais.

2. Marie-Andrée Roy est professeure au Département des sciences religieuses et à l'Institut de recherches et d'études féministes (IREF) de l'UQAM, Lyne Kurtzman est chercheure à l'Alliance de recherche IREF/Relais-femmes à l'UQAM.

3. Le rêve brisé de Cendrillon, Grands reportages, RDI, Production Mira 2002.

4. Ce texte est une traduction libre du texte du prophète Jérémie 31,15 .

5. La sculpture Crucified Woman est l'œuvre de Almuth Lutkenhaus-Lackey, elle a été terminée en 1976. En 1979, elle a d'abord été suspendue dans le chœur d'une église de l'Église Unie sur la rue Bloor à Toronto, puis elle a été donnée au Emmanuel College. À la suite de controverses dans ces deux endroits, elle a finalement été placée dans un jardin du campus de l'Université de Toronto. 